\title{
Core Decompression Combined with Implantation of Autologous Bone Marrow Concentrate with Tricalcium Phosphate Does Not Prevent Radiographic Progression in Early Stage Osteonecrosis of the Hip
}

\author{
Alejandro Hernandez, PhD, Jorge H. Nuñez, MD, Andrea Sallent, MD, Albert Gargallo-Margarit, MD,
} Irene Gallardo-Calero, MD, Victor Barro, MD

Hip Unit, Department of Orthopedic Surgery, University Hospital of Vall d'Hebron, Universitat Autónoma de Barcelona, Barcelona, Spain

Background: Untreated osteonecrosis of the femoral head ultimately leads to secondary coxarthrosis. The aim of this study was to determinate if the core decompression of the femoral head combined with implantation of autologous bone marrow concentrate with tricalcium phosphate could be used to prevent radiographic progression of early stage osteonecrosis of the hip. We also sought to determine whether this treatment improved clinical outcomes and reduced the need for total hip arthroplasty.

Methods: Eighteen hips were included in the present study. All of them underwent core decompression of the femoral head combined with implantation of autologous bone marrow concentrate with tricalcium phosphate between 2007 and 2012. The cell concentrate was obtained from the posterior iliac crest and processed and implanted during the same surgical procedure. Patient demographic data, clinical data including modified Harris hip score, and radiological data were collected preoperatively, postoperatively, and during the follow-up period. Also, survival endpoints were analyzed: time of femoral head collapse and need for total hip arthroplasty.

Results: The mean age of patients was 37.8 years (standard deviation [SD], 9.31 years). The mean follow-up was 68.9 months (SD, 15.0 months). In most cases (70.6\%), the etiology of the osteonecrosis of the femoral head was corticosteroid use; in the remaining cases, secondary to alcohol use. Core decompression of the femoral head combined with implantation of autologous bone marrow concentrate with tricalcium phosphate did not prevent progression to collapse $(<80 \%$ at 5 years) although modified Harris hip scores improved. Overall median survival with the total hip arthroplasty as endpoint was 23 months ( $95 \%$ confidence interval [Cl], 14.9 to 31.1 months). Overall median survival time with any degree of collapse as endpoint was 7 months $(95 \% \mathrm{Cl}, 2.0$ to 12.0 months).

Conclusions: The results obtained in this study suggest that core decompression combined with implantation of autologous bone marrow concentrate and tricalcium phosphate will not prevent radiographic progression of early stage osteonecrosis of the hip. These finding also suggest that the absence of indications for hip replacement alone is not an indicator of good response to the treatment, and it is important to note the radiological results.

Keywords: Hip, Osteonecrosis, Orthopedic procedures, Bone marrow, Bone substitutes

Received March 11, 2019; Accepted September 3, 2019

Correspondence to: Jorge H. Nuñez, MD

Hip Unit, Department of Orthopedic Surgery, University Hospital of Vall d'Hebron, Universitat Autónoma de Barcelona, Barcelona 08035, Spain Tel: +34-934893000, Fax: +34-934894401, E-mail: j.nunez@vhebron.net

Copyright (C) 2020 by The Korean Orthopaedic Association 
Osteonecrosis of the femoral head (ONFH) commonly affects patients in the third to fifth decades of life. ${ }^{1)}$ Although the ultimate goal of treatment of ONFH is preservation of the femoral head to avoid a total hip arthroplasty (THA), this pathology accounts for up to $46.9 \%$ of THAs in these age groups. ${ }^{2,3)}$ Moreover, more than $50 \%$ of patients will develop bilateral ONFH. ${ }^{3)}$

Although numerous risk factors for ONFH have been identified (e.g., corticosteroids, alcohol use, sicklecell disease, Caisson disease, dyslipidemia, and coagulation disorders), the etiology and pathogenesis of this disease remain unclear. ${ }^{2,4)}$ ONFH permanently alters the bone mass, producing a weak mineral structure that tends to collapse the femoral head and destroy the hip joint. ${ }^{5)}$ Although some nonsurgical treatment modalities have been reported, surgery is the standard treatment approach. However, the optimal surgical method to preserve the femoral head remains unclear. ${ }^{1,2}$

Various hip preserving procedures have been described, including the standard core decompression of the femoral head $(\mathrm{CDFH})$, osteotomies, and bone grafting (vascularized or nonvascularized) ${ }^{2,6,7)}$ Emerging techniques include CDFH combined with instillation of mesenchymal stem cells (MSC) of autologous bone marrow and growth factors applied to the necrotic area. ${ }^{8,9)}$ The rationale underlying this combined approach is the decrease in the number and quality of stem cells in the bone marrow of patients with ONFH. ${ }^{10-12)}$ However, to date, none of these treatments that aim to delay the need for THA has produced significantly superior outcomes compared to core decompression alone. ${ }^{2,8)}$ Moreover, the fact that $\mathrm{CDFH}$ is done with a variety of techniques and with supplemental bone graft procedures suggests that surgeons have not been completely satisfied with the results. ${ }^{6}$

In this context, the aim of the present study was to determine if CDFH and implantation of autologous bone marrow concentrate with tricalcium phosphate could prevent radiographic progression in patients with early stage hip osteonecrosis. Secondarily, we sought to determine if this treatment approach improves clinical outcomes and decreases the need for THA.

\section{METHODS}

A case series study of patients with a diagnosis of ONFH was performed between 2009 and 2014. The study was approved by the hospital ethics committee (IRB No. IDRTF055), and all patients gave their informed consent prior to inclusion in the study. The inclusion criteria were as follows: (1) patients aged $>18$ and $<45$ years; (2) patients with ONFH; and (3) patients with Association Research Circulation Osseous (ARCO) classification stages I or II confirmed by magnetic resonance imaging (MRI). The following exclusion criteria were used: (1) patients with previous surgery in the hip; (2) patients with previous medical treatments for ONFH; and (3) patients with a follow-up less than 2 years.

\section{Surgical Technique}

The same hip orthopedic surgery specialist $(\mathrm{AH})$ performed all operations. One hour before the core decompression operation, the patient was turned into a prone position and $60 \mathrm{~mL}$ of bone marrow was aspirated from the posterosuperior iliac spine on both sides (Fig. 1). For 15 minutes, the bone marrow was centrifuged to collect buffy coat by using a cell separator at Hematology Department Laboratory. From the $60 \mathrm{~mL}$ of bone marrow, we obtained a concentrate of $10 \mathrm{~mL}$ of progenitor stem cells. After the concentrated marrow was sent back to the operating room, $\mathrm{CDFH}$ was performed by drilling, under fluoroscopic guidance, with a $2-\mathrm{mm}$ threaded needle. The needle was placed from the lateral cortex of the femur to the center of the necrotic area.

Next, an 8-mm drill bit was used to drill the cortex and medulla and a spoon-shaped curette was used to complete the curettage of necrotic bone. Then, through the drill hole, the patient was injected with the concentrated bone marrow aspirate and tricalcium phosphate matrix (Cellplex 100-400- $\mu$ m porosity; Wright Medical Technology, Arlington, TN, USA) (Fig. 2). After the injection, the entrance hole was obliterated by directing the core needle tangentially to collapse the core hole by cancellous bone and prevent leakage of the combined concentrate. Postoperatively, all patients started the same physical rehabilitation program. Six weeks of non-weight-bearing with bilateral crutches was advised to all patients. Patients were allowed to engage in physical activities and sports 6

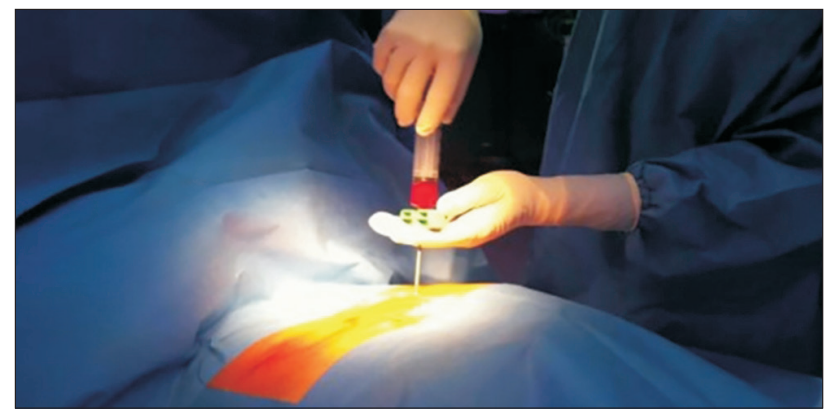

Fig. 1. The patient was placed in prone position, and 60-mL bone marrow was aspirated from the posterosuperior iliac spine on both sides. 

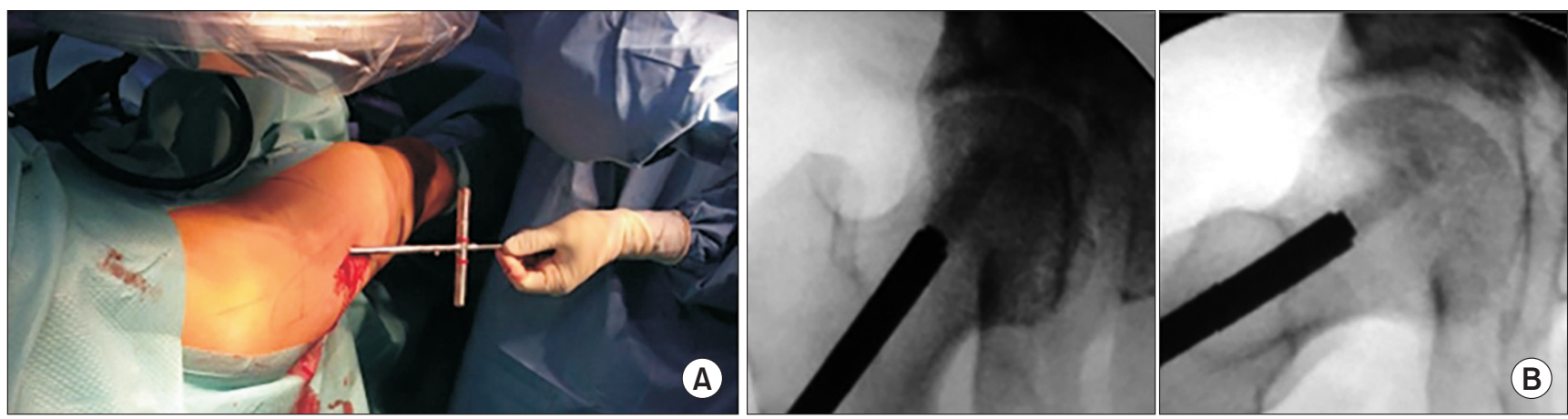

Fig. 2. (A) Core decompression of the femoral head was performed by drilling, under fluoroscopic guidance, with a 2-mm threaded needle. Next, an 8-mm drill bit was used to drill the cortex and medulla to complete curettage of the necrotic bone. (B) Through the drill hole, the patient was injected with the concentrated bone marrow aspirate and tricalcium phosphate matrix (Cellplex 100-400-um porosity; Wright Medical Technology, Arlington, TN, USA) under fluoroscopic guidance.
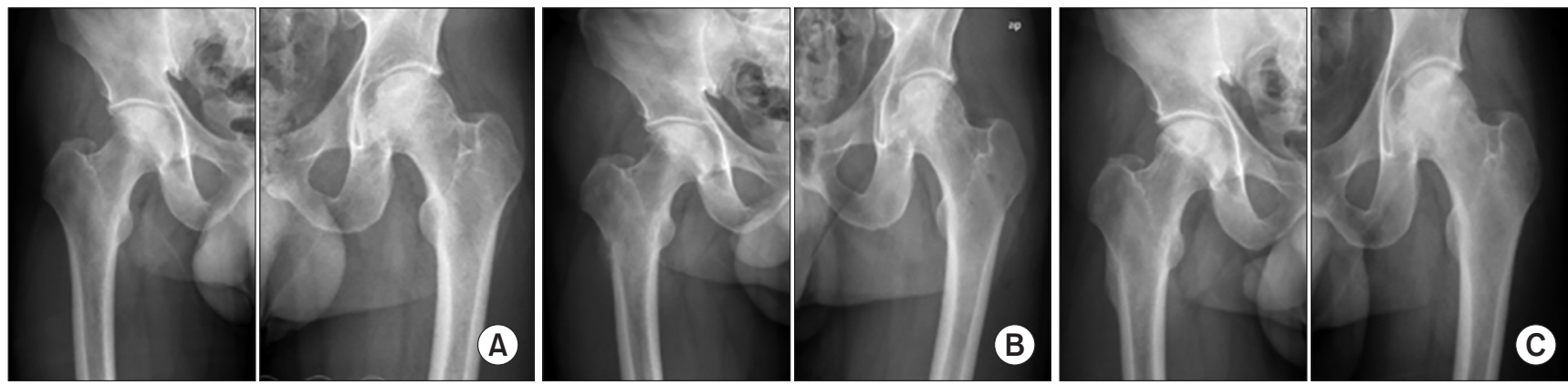

Fig. 3. (A) Preoperative anteroposterior hip radiograph. (B) One-month postoperative anteroposterior hip radiograph. (C) Twelve-month postoperative anteroposterior hip radiograph.

months after surgery.

\section{Follow-up and Outcome Measures}

Demographic, clinical, and radiological data were collected preoperatively, postoperatively, and during the follow-up period $(1,3,6$, and 12 months postoperatively and every 6 months thereafter). The clinical data from 24 months of follow-up from each patient were used for analysis. All scores were measured by the same observer (AS). Demographic and clinical data included the patient's age at the time of surgery, sex, etiology of the ONFH, ARCO classification of the ONFH, and modified Harris hip score (mHHS). Radiological analysis included radiographic (preoperative and every postoperative follow-up) and MRI evaluation (preoperative, 6 months postoperative, and every 12 months thereafter). During follow-up, the radiologic increase of the lesion, measured by the Kerboul angle, and the development of a femoral head collapse were evaluated by a single expert radiologist (RD) in musculoskeletal pathology (Fig. 3). Collapse of the femoral head was considered to have occurred if subchondral fracture (crescent fracture) or loss of femoral head morphology was observed in radiographic or MRI evaluation.

\section{Statistical Analysis}

Statistical analysis was conducted using IBM SPSS ver. 20.0 (IBM Corp., Armonk, NY, USA). Descriptive statistics were used to present the results by mean or median and range. Pre- and postoperative mHHS was compared by using paired $t$-tests. Differences with $p$-values $<0.05$ were considered statistically significant. The Kaplan-Meier estimator was used to determine survival. The survival analysis was based on two endpoints: time to femoral head collapse and time to THA.

\section{RESULTS}

There were 20 hips in 13 patients who underwent CDFH combined with implantation of autologous bone marrow concentrate with tricalcium phosphate. However, two patients were excluded from the study because they were lost in the follow-up and one patient was excluded because he 
Hernandez et al. Bone Marrow Concentrate with Tricalcium Phosphate in Hip Osteonecrosis

Clinics in Orthopedic Surgery • Vol. 12, No. 2, $2020 \bullet$ www.ecios.org

developed a subtrochanteric fracture after surgery, requiring osteosynthesis with an intramedullary nail. It is worth noting that these patients did not develop femoral head collapse while they were followed up. The mean age of the final sample (10 patients, 17 hips) was 37.8 years (standard deviation [SD], 9.3 years). There were seven men and three women. The mean follow-up was 68.9 months (SD, 15.0 months) (Table 1 ).

In most cases (12 of 17 hips), the etiology of ONFH was corticosteroid use; in the remaining five cases, sec-

\section{Table 1. Patient Demographics and Clinical Data}

\begin{tabular}{lccc}
\multicolumn{1}{c}{ Variable } & $\begin{array}{c}\text { Total } \\
(\mathrm{N}=10)\end{array}$ & $\begin{array}{c}\text { ARC0 stage I } \\
(\mathrm{n}=6)\end{array}$ & $\begin{array}{c}\text { ARCO stage II } \\
(\mathrm{n}=4)\end{array}$ \\
\hline Sex & $7(70)$ & $4(66.6)$ & $3(75)$ \\
Male & $3(30)$ & $2(33.4)$ & $1(25)$ \\
\hline Female & $37.8 \pm 9.3$ & $37.2 \pm 9.1$ & $38.1 \pm 9.6$ \\
\hline Age (yr) & & & \\
\hline Etiology & $6(60)$ & $4(66.6)$ & $2(50)$ \\
\hline Corticosteroid use & $4(40)$ & $2(33.4)$ & $2(50)$ \\
\hline Alcohol use & & & \\
\hline Modified Harris hip score & & & \\
\hline Preoperative & $76.7 \pm 9.8$ & $78.2 \pm 9.1$ & $74.6 \pm 10.1$ \\
\hline Postoperative & $85.2 \pm 11.4$ & $86.2 \pm 12.4$ & $84.3 \pm 10.9$ \\
\hline Follow-up (mo) & $68.9 \pm 15.0$ & $68.7 \pm 14.2$ & $70.5 \pm 13.8$ \\
\hline
\end{tabular}

Values are presented as number (\%) or mean \pm standard deviation. ARCO: Association Research Circulation Osseous.

\begin{tabular}{|c|c|c|}
\hline Subclassification & Kerboul angle $\left({ }^{\circ}\right)$ & Number \\
\hline Total & Total & 17 \\
\hline \multirow[t]{4}{*}{ Central } & I (<200) & 0 \\
\hline & II (200-249) & 2 \\
\hline & III (250-299) & 3 \\
\hline & IV $(>300)$ & 0 \\
\hline \multirow[t]{4}{*}{ Lateral } & I (<200) & 1 \\
\hline & II (200-249) & 4 \\
\hline & III (250-299) & 2 \\
\hline & IV (>300) & 5 \\
\hline
\end{tabular}

ondary to alcohol use. By ARCO stage, 11 hips were classified as stage I and six hips, as stage II. The size of femoral necrotic lesion was measure by the Kerboul angle (Table 2). Edema in MRI of the femoral head was a usual sign in our patients. The mean postoperative mHHS at 24 months of follow-up was 85.2 (SD, 11.4). The mHHS at 24 months of follow-up improved statistically significantly $(p<0.05)$ (Table 1).

Nevertheless, 15 of 17 hips progressed to ARCO stage III, and they underwent collapse or subchondral fracture during the follow-up. Nine of 15 hips underwent THA due to severe pain or disability. The probability of not presenting femoral head collapse in the first 15 months was $33 \%$. The overall median survival time (with THA as endpoint) was 23 months (95\% confidence interval [CI], 14.9 to 31.1 months) (Fig. 4). Overall median survival time for any degree of collapse or progression to stage III

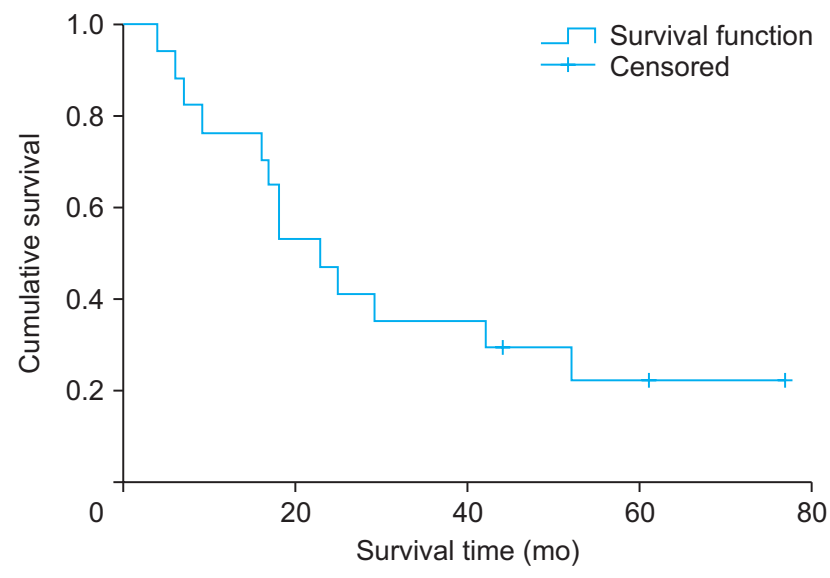

Fig. 4. Median 5-year survival time with total hip arthroplasty as an endpoint.

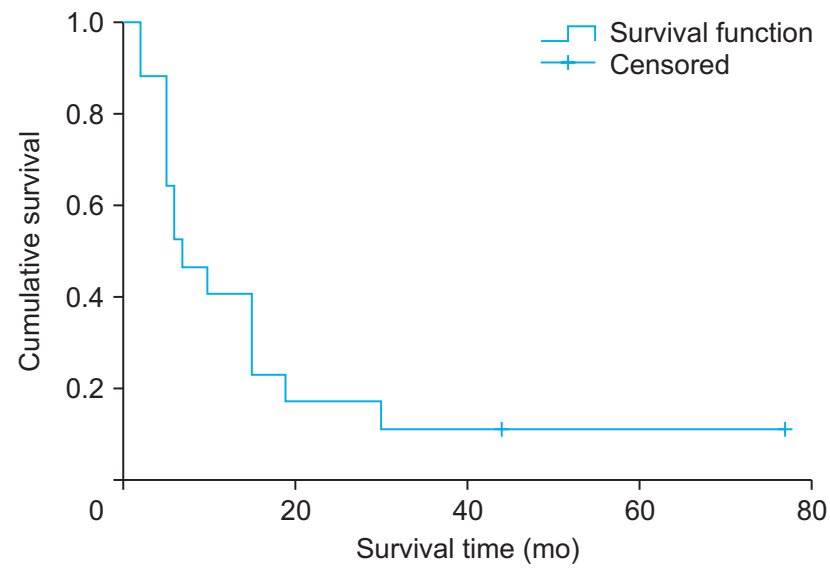

Fig. 5. Median 5-year survival time with collapse of the femoral head as an endpoint. 
Hernandez et al. Bone Marrow Concentrate with Tricalcium Phosphate in Hip Osteonecrosis

Clinics in Orthopedic Surgery • Vol. 12, No. 2, $2020 \bullet$ www.ecios.org

disease was 7 months (95\% CI, 2.0 to 12.0 months) (Fig. 5).

\section{DISCUSSION}

ONFH is a devastating condition affecting relatively young patients whereby the femoral head is necrotic, resulting in significant pain, articular surface collapse, and eventual osteoarthritis. ${ }^{1-3)}$ The main aim of the present study was to determine if $\mathrm{CDFH}$ with adjuvant implantation of autologous bone marrow concentrate and tricalcium phosphate could prevent radiographic progression of early stage osteonecrosis of the hip. We also sought to determine whether this treatment improved clinical outcomes and reduced the need for THA. The overall median survival for the two study endpoints-need for THA and femoral head collapse-were 23 months and 7 months, respectively.

We used autologous bone marrow concentrate because previous studies have suggested that treatment results of ONFH are better in patients who receive implantation of bone marrow concentrate than those who do not. ${ }^{13)}$ In our study, we combined the autologous bone marrow concentrate with tricalcium phosphate-a synthetic biocompatible, resorbable, osteoconductive material-to improve defect filling, cell transportation, and mechanical structural support, all of which are considered to play an essential role after core decompression and during revascularization. ${ }^{14)}$ Most previous studies have evaluated the outcomes of CDFH with supplementation of stem cells and growth factors but without the use of porous bone filler. ${ }^{2,15,16)}$ The first authors who described this technique with porous bone filler were Liu et al., ${ }^{17)}$ who retrospectively analyzed the effectiveness of core decompression and implantation of porous hydroxyapatite bone filler with or without autologous bone marrow concentrate for the treatment ONFH. At a mean follow-up of 26 months, they found that the collapse rate was lower in the group that received autologous bone marrow concentrate than the group that did not receive this adjuvant treatment (17.5\% vs. 59.3\%). ${ }^{17)}$ Yamasaki et al. ${ }^{18)}$ reported a $10 \%$ collapse rate after 18 months of follow-up in patients treated with a combination of hydroxyapatite particles and autologous bone marrow concentrate versus a $75 \%$ collapse rate in patients treated with hydroxyapatite particles alone. In a similar study, Kang et al. ${ }^{19)}$ used an autologous iliac crest graft associated with MSCs to fill the debrided cavity in 61 hips, reporting a $65.7 \%$ success rate (clinical and radiological results) in stage II patients; however, the collapse rate was not specified.

In the present study, we found that performing $\mathrm{CDFH}$, debridement, and implantation of autologous bone marrow concentrate embedded in tricalcium phosphate did not prevent femoral head collapse in most cases $(>80 \%)$ at 5-year follow-up. The treatment failure rate in the present study was higher than the rates reported in some studies $(6 \%-25 \%){ }^{16,20,21)}$ However, we are not the first to report such high failure rates. For example, Chotivichit et al. ${ }^{22)}$ evaluated 13 cases of stage I or II ONFH and found radiological progression indicative of femoral head collapse in $72 \%$ of the patients at a mean follow-up of 3.6 years. We believe that the high failure rate in our study can be explained by the presence of important, ongoing risk factors such as steroid use and alcohol consumption. In the follow-up, the steroid treatment and alcohol consumption were stopped in our patients. Additionally, the use of MRI, which is more sensitive and specific than standard radiography, to assess the increase in the lesion size and the development of femoral head collapse could have led to higher failure rates given that several of the aforementioned studies with better results did not use MRI., ${ }^{3,16,17)}$ In those studies, they used X-ray in the follow-up, which is an important limitation. Indeed, we chose to use MRI because of its high sensitivity and specificity, which is why MRI is considered the benchmark for the diagnosis and follow-up of osteonecrosis. ${ }^{2)}$ Another important difference between our study and other studies that also used autologous bone marrow concentrate with porous bone filler is that those studies had substantially shorter follow-up periods $(<3$ years $) .{ }^{17,18)}$ In our study, we had a minimum follow-up of 5 years.

The disparity between our findings and other studies could also be related to the surgical technique used for CDFH. In our case, the diameter of the drill hole was $8 \mathrm{~mm}$ in all patients. The number and diameter of drillings can vary widely depending on the specific $\mathrm{CDFH}$ technique. ${ }^{8,23,24)}$ For that reason, the number and diameter of the drillings determine the bone marrow concentrate implantation method in the necrotic area. ${ }^{19,20,25,26)} \mathrm{Al}-$ though some authors postulate that multiple small-diameter drillings are superior to the classic $8-10 \mathrm{~mm}$ drillings, there is no clear evidence regarding the superiority of that approach. ${ }^{8,23,24,27,28)}$ Some studies have suggested that small-diameter multichannel core decompression is suitable for pre-collapsed lesions. ${ }^{15)}$ For example, Lim et al. ${ }^{27)}$ compared a CDFH technique using large-diameter drillings, curettage, and bone graft versus multiple 3-mm perforations and autologous bone marrow concentrate implantation, reporting no statistically significant differences between the two techniques at the 5-year follow-up. At our center, we prefer large-diameter core decompression because it offers two important advantages: high pressure 
within the necrotic femoral head could achieve sufficient decompression, and progressively expanding reamers can be used to completely remove the sequestrum and fatty marrow within the necrotic area.

It is worth noting that clinical success did not correspond closely with radiographic success in this study. Even though some of our patients experienced radiographic progression, their mHHS at 24 months of followup significantly improved, as was previously reported by Baksi et al. ${ }^{29)}$ and Sallam et al. ${ }^{3)}$ We think that this could be because even in stage I and II, the femoral head remains without collapse and the pain is related to edema and high bone pressure. Also, the size of the necrotic lesion was large (200 or more degrees) in 16 of the 17 hips evaluated. In our study, the median survival time (defined as time until THA) was 23 months. Nine of the patients eventually required a THA during follow-up. Despite the encouraging clinical results and lower THA conversion rates reported in the literature with bone marrow concentration, it is important to note that the studies that had longer followup times (including ours) have found a higher rate of radiological progression of femoral head collapse as time passes. ${ }^{9,16)}$ This is important because prosthetic surgery in hip joint arthrosis does not rely solely on the degree of radiological involvement. In other words, although hip replacement may not be indicated, this does not imply a good treatment response.

This study has several limitations. First, the sample size was relatively small mainly due to the low incidence of detecting ONFH in the ARCO stages I and II, which is consistent with other published studies (between $0 \%$ and $2 \%)$. Another potential limitation is that we did not have a control group. Despite these limitations, this study has also several unique attributes. First, to our knowledge, our study presents the longest follow-up (> 68 months) after core decompression and autologous bone marrow concen- trate implantation with porous material for the treatment of ONFH. Second, MRI was used in the follow-up. We recommend that future studies should be long-term prospective studies that involve a large sample size. Moreover, they should compare and evaluate different surgical treatments including the use of different porous materials (such as hydroxyapatite composite bone) used for the treatment of this pathology.

In our study, we found that core decompression combined with implantation of autologous bone marrow concentrate and tricalcium phosphate did not prevent radiographic progression of early stage osteonecrosis of the hip. We found that it did not prevent progression of disease to collapse ( $<80 \%$ at 5 years) although Harris hip scores improved. These findings suggest that the absence of indications for hip replacement alone is not an indicator of good response to the treatment, and it is important to note radiological results.

\section{CONFLICT OF INTEREST}

No potential conflict of interest relevant to this article was reported.

\section{ACKNOWLEDGEMENTS}

We would like to thank the Department of Radiology of University Hospital of Vall d' Hebron, Barcelona, Spain. It provided all hip computer tomographic images of the patients included in our study.

We would like to thank Rosa Dominguez-Oronoz (Department of Radiology, University Hospital of Vall d' Hebron, Barcelona, Spain), who was the radiologist who analyzed all hip magnetic resonance images of the patients included in our study.

\section{REFERENCES}

1. Rajpura A, Wright AC, Board TN. Medical management of osteonecrosis of the hip: a review. Hip Int. 2011;21(4):38592.

2. Zalavras CG, Lieberman JR. Osteonecrosis of the femoral head: evaluation and treatment. J Am Acad Orthop Surg. 2014;22(7):455-64.

3. Sallam AA, Imam MA, Salama KS, Mohamed OA. Inverted femoral head graft versus standard core decompression in nontraumatic hip osteonecrosis at minimum 3 years followup. Hip Int. 2017;27(1):74-81.
4. Mont MA, Jones LC, Hungerford DS. Nontraumatic osteonecrosis of the femoral head: ten years later. J Bone Joint Surg Am. 2006;88(5):1117-32.

5. Mankin HJ. Nontraumatic necrosis of bone (osteonecrosis). N Engl J Med. 1992;326(22):1473-9.

6. Amanatullah DF, Strauss EJ, Di Cesare PE. Current management options for osteonecrosis of the femoral head: part II, operative management. Am J Orthop (Belle Mead NJ). 2011;40(10):E216-25.

7. Ficat RP. Idiopathic bone necrosis of the femoral head. Early 
Hernandez et al. Bone Marrow Concentrate with Tricalcium Phosphate in Hip Osteonecrosis

Clinics in Orthopedic Surgery • Vol. 12, No. 2, $2020 \bullet$ www.ecios.org

diagnosis and treatment. J Bone Joint Surg Br. 1985;67(1):3 9.

8. Marker DR, Seyler TM, Ulrich SD, Srivastava S, Mont MA. Do modern techniques improve core decompression outcomes for hip osteonecrosis? Clin Orthop Relat Res. 2008;466(5):1093-103.

9. Hernigou P, Poignard A, Zilber S, Rouard H. Cell therapy of hip osteonecrosis with autologous bone marrow grafting. Indian J Orthop. 2009;43(1):40-5.

10. Gangji V, Hauzeur JP, Schoutens A, Hinsenkamp M, Appelboom T, Egrise D. Abnormalities in the replicative capacity of osteoblastic cells in the proximal femur of patients with osteonecrosis of the femoral head. J Rheumatol. 2003;30(2):348-51.

11. Hernigou P, Beaujean F, Lambotte JC. Decrease in the mesenchymal stem-cell pool in the proximal femur in corticosteroid-induced osteonecrosis. J Bone Joint Surg Br. 1999;81(2):349-55.

12. Gangji V, Hauzeur JP, Matos C, De Maertelaer V, Toungouz M, Lambermont M. Treatment of osteonecrosis of the femoral head with implantation of autologous bone-marrow cells: a pilot study. J Bone Joint Surg Am. 2004;86(6):115360.

13. Rastogi S, Sankineani SR, Nag HL, et al. Intralesional autologous mesenchymal stem cells in management of osteonecrosis of femur: a preliminary study. Musculoskelet Surg. 2013;97(3):223-8.

14. Brown TD, Pedersen DR, Baker KJ, Brand RA. Mechanical consequences of core drilling and bone-grafting on osteonecrosis of the femoral head. J Bone Joint Surg Am. 1993;75(9):1358-67.

15. Lieberman JR, Conduah A, Urist MR. Treatment of osteonecrosis of the femoral head with core decompression and human bone morphogenetic protein. Clin Orthop Relat Res. 2004;(429):139-45.

16. Gangji V, De Maertelaer V, Hauzeur JP. Autologous bone marrow cell implantation in the treatment of non-traumatic osteonecrosis of the femoral head: five year follow-up of a prospective controlled study. Bone. 2011;49(5):1005-9.

17. Liu Y, Liu S, Su X. Core decompression and implantation of bone marrow mononuclear cells with porous hydroxylapatite composite filler for the treatment of osteonecrosis of the femoral head. Arch Orthop Trauma Surg. 2013;133(1):12533.

18. Yamasaki T, Yasunaga Y, Ishikawa M, Hamaki T, Ochi M. Bone-marrow-derived mononuclear cells with a porous hydroxyapatite scaffold for the treatment of osteonecrosis of the femoral head: a preliminary study. J Bone Joint Surg Br. 2010;92(3):337-41.

19. Kang JS, Moon KH, Kim BS, et al. Clinical results of autoiliac cancellous bone grafts combined with implantation of autologous bone marrow cells for osteonecrosis of the femoral head: a minimum 5-year follow-up. Yonsei Med J. 2013;54(2):510-5.

20. Hernigou P, Beaujean F. Treatment of osteonecrosis with autologous bone marrow grafting. Clin Orthop Relat Res. 2002;(405):14-23.

21. Wang BL, Sun W, Shi ZC, et al. Treatment of nontraumatic osteonecrosis of the femoral head with the implantation of core decompression and concentrated autologous bone marrow containing mononuclear cells. Arch Orthop Trauma Surg. 2010;130(7):859-65.

22. Chotivichit A, Korwutthikulrangsri E, Auewarakul C, Sarirasririd S. Core decompression and concentrated autologous bone marrow injection for treatment of osteonecrosis of the femoral head. J Med Assoc Thai. 2012;95 Suppl 9:S14-20.

23. Mont MA, Ragland PS, Etienne G. Core decompression of the femoral head for osteonecrosis using percutaneous multiple small-diameter drilling. Clin Orthop Relat Res. 2004;(429):131-8.

24. Song WS, Yoo JJ, Kim YM, Kim HJ. Results of multiple drilling compared with those of conventional methods of core decompression. Clin Orthop Relat Res. 2007;454:139-46.

25. Wang T, Wang W, Yin ZS. Treatment of osteonecrosis of the femoral head with thorough debridement, bone grafting and bone-marrow mononuclear cells implantation. Eur J Orthop Surg Traumatol. 2014;24(2):197-202.

26. Gangji V, Hauzeur JP. Treatment of osteonecrosis of the femoral head with implantation of autologous bone-marrow cells. Surgical technique. J Bone Joint Surg Am. 2005;87 Suppl 1(Pt 1):106-12.

27. Lim YW, Kim YS, Lee JW, Kwon SY. Stem cell implantation for osteonecrosis of the femoral head. Exp Mol Med. 2013;45:e61.

28. Sen RK, Tripathy SK, Aggarwal S, Marwaha N, Sharma RR, Khandelwal N. Early results of core decompression and autologous bone marrow mononuclear cells instillation in femoral head osteonecrosis: a randomized control study. J Arthroplasty. 2012;27(5):679-86.

29. Baksi DP, Pal AK, Baksi DD. Long-term results of decompression and muscle-pedicle bone grafting for osteonecrosis of the femoral head. Int Orthop. 2009;33(1):41-7. 